\title{
Uso de medicamentos como fator de risco para fratura grave decorrente de queda em idosos
}

\author{
Medication as a risk factor for falls resulting \\ in severe fractures in the elderly
}

Evandro da Silva Freire Coutinho 1,2

Sidney Dutra da Silva 3

\footnotetext{
1 Departamento de Epidemiologia e Métodos Quantitativos em Saúde, Escola Nacional de Saúde Pública, Fundação Oswaldo Cruz.

Rua Leopoldo Bulhões 1480, Rio de Janeiro, $R J$ 21041-210, Brasil.

2 Instituto de Medicina Social, Universidade do Estado do Rio de Janeiro. Rua São Francisco Xavier 524, Rio de Janeiro, $R J$ 20550-013, Brasil.

3 Universidade Aberta da Terceira Idade, Universidade do Estado do Rio de Janeiro. Rua São Francisco Xavier 524, Rio de Janeiro, $R J$ 20550-013, Brasil.
}

\begin{abstract}
Falls leading to fractures among the elderly are a major public problem. A case-control study was conducted on the use of certain drugs as a risk factor for hospitalization due to fractures after falls among individuals aged 60 years or over in the city of Rio de Janeiro, Brazil. One hundred sixty-nine cases and 315 in-patient controls were matched by age, sex, and hospital. Odds ratios (OR) adjusted for potential confounders were calculated using conditional logistic regression. Increased risk of such accidents was found for calcium channel antagonists $(O R=$ 1.96, 1.16-3.30) and benzodiazepines (OR =2.09, 1.08-4.05), and decreased risk was associated with diuretics $(O R=0.40,0.20-0.80)$. Antacids, digitalis, and laxatives were associated with reduced risk of fractures after falls that reached borderline statistical significance $(0.05<p<0.10)$. The findings highlight the need to weigh risks and benefits of medication in the elderly. It is also important to advise such individuals and their families on how to avoid falls when such medication proves necessary.
\end{abstract}

Key words Fractures; Drugs; Aging Health; Accidental Falls

Resumo Quedas seguidas de fraturas entre idosos constituem um importante problema de saúde pública. Um estudo caso-controle foi conduzido para avaliar o papel do uso de um conjunto de medicamentos, como fator de risco para esses acidentes entre pessoas com 60 anos ou mais, na cidade do Rio de Janeiro, Brasil. Cento e sessenta e nove casos de internação por fratura conseqüente a queda, e 315 controles hospitalares foram pareados por idade, sexo e hospital. Odds ratios (OR), ajustados por fatores potenciais de confusão, foram obtidos utilizando-se regressão logística condicional. Observou-se um aumento no risco desses acidentes para o uso de drogas bloqueadoras dos canais de cálcio $(O R=1,96,1,16-3,30)$ e benzodiazepínicos $(O R=2,09,1,08$ 4,05), e uma redução para o uso de diuréticos (OR=0,40,0,20-0,80). Antiácidos, digitálicos e laxantes mostraram-se associados a uma redução do risco de fraturas por quedas, cuja significância estatística atingiu níveis limítrofes $(0,05<p<0,10)$. Os dados deste estudo apontam para a necessidade de ponderar os riscos e benefícios no uso de medicamentos em idosos, e orientar tais indivíduos e seus familiares para evitar esses acidentes quando o uso desses medicamentos é necessário.

Palavras-chave Fraturas; Medicamentos; Saúde do Idoso; Acidentes por Quedas 


\section{Introdução}

Assim como muitos países do Terceiro Mundo, o Brasil vem experimentando um importante crescimento de sua população de idosos (Kalache et al., 1987). No país, sobretudo em algumas regiões, experimenta-se um processo de envelhecimento populacional de intensidade comparável àquela observada em países do Primeiro Mundo. Desde 1940, o grupo etário com 60 anos ou mais é aquele que, proporcionalmente, mais tem crescido (Monteiro \& Alves, 1995).

As quedas são reconhecidas como um importante problema de saúde pública entre os idosos, em decorrência da freqüência, da morbidade e do elevado custo social e econômico decorrente das lesões provocadas. Estudos realizados nos Estados Unidos e na Europa mostram que aproximadamente um terço da população acima de 65 anos sofreu pelo menos uma queda durante o último ano. Entre idosos que sofreram quedas, 3-5\% apresentaram fraturas graves, isto é, que demandaram internação hospitalar (Ryynänen et al., 1991; Sattin, 1992; Tinetti, 1994).

No Brasil, Chaimowicz et al. (2000) observaram uma incidência de $17 \%$ de pelo menos uma queda no ano anterior, entre os idosos que residiam num bairro do Município de Campo Belo, Minas Gerais. Em outro estudo, conduzido com a população de idosos que freqüentava a Universidade Aberta da Terceira Idade (UnATI), da Universidade do Estado do Rio de Janeiro (UERJ), Rozenfeld (1997) encontrou uma incidência de $38 \%$ de quedas nos 12 meses anteriores à entrevista.

Pinheiro (1999) conduziu um estudo sobre as internações de pacientes com diagnóstico principal de fratura de colo de fêmur (total de 1.870 internações), nos hospitais credenciados pelo Sistema Único de Saúde (SUS), da cidade do Rio de Janeiro, nos anos de 1994 e 1995. A média de idade do grupo tratado clinicamente foi de 61 anos, e no grupo tratado cirurgicamente de 68,8 anos. O tempo médio de permanência (TMP), foi de 10,6 dias no grupo de pacientes tratados clinicamente, e de 16,2 dias no grupo de pacientes que foram submetidos à cirurgia. O TMP nos hospitais pesquisados variou entre 5,3 e 34,7 dias.

As quedas, além de produzirem uma importante perda de autonomia e de qualidade de vida entre os idosos, podem ainda repercutir entre os seus cuidadores, principalmente os familiares, que devem se mobilizar em torno de cuidados especiais, adaptando toda a rotina em função da recuperação ou adaptação após a queda.
O uso de medicamentos tem sido abordado em vários estudos como um dos fatores de risco para tais acidentes. Leipzig et al. (1999a, 1999b) realizaram uma meta-análise com estudos que investigaram o papel de medicamentos psiquiátricos, cardiológicos e analgésicos sobre o risco de quedas entre idosos. De acordo com esse estudo, benzodiazepínicos, neurolépticos, sedativos/hipnóticos, antidepressivos, diuréticos em geral, antiarrítimicos e digoxina associaram-se ao maior risco de quedas na população acima de 60 anos.

Tinetti (1994), em sua proposta de agenda de pesquisa para a prevenção de lesões decorrentes de queda em idosos, assinala a importância da identificação dos fatores de risco para a redução da incidência de tais acidentes. Ainda segundo a autora, embora muitos estudos tenham sido realizados para identificar fatores de risco para quedas, poucos se ocuparam dos determinantes das lesões importantes decorrentes desses acidentes. Em nosso meio, só identificamos dois estudos que investigaram o papel do uso de medicamentos sobre o risco de quedas, sem se preocupar com a ocorrência ou não de fraturas severas.

Neste artigo, apresentamos dados sobre o papel do uso de medicamentos no risco de fraturas decorrentes de quedas, que demandam internação hospitalar (fraturas graves) entre idosos da população geral.

\section{Material e métodos}

\section{Desenho}

Realizou-se um estudo caso-controle de base secundária. Para cada caso foram selecionados dois controles, pareados individualmente por sexo, idade e hospital.

\section{Fonte de casos e controles}

Os casos foram indivíduos de ambos os sexos, com 60 ou mais anos de idade, residentes no Município do Rio de Janeiro, internados por fratura decorrente de queda em cinco hospitais públicos da cidade: Hospital Universitário Clementino Fraga Filho, Hospital Municipal Miguel Couto, Hospital Municipal Salgado Filho, Hospital do Andaraí e Hospital Universitário Pedro Ernesto. Os controles foram selecionados nos mesmos hospitais, entre pacientes cuja patologia que determinou a internação não estava associada com o uso dos medicamentos de interesse. 


\section{Coleta de dados}

As entrevistas foram realizadas utilizando-se um questionário contendo perguntas sobre dados sócio-demográficos, condição de saúde, morbidade auto-referida, grau de autonomia, riscos domiciliares, circunstâncias da queda, ocorrência de quedas e fraturas no ano anterior, uso de substâncias - álcool, café, tabaco e medicamentos no período em que se deu a queda -, além de uma escala para deficiência cognitiva - BOAS - (Veras \& Coutinho, 1991). No caso do idoso não ter condição de responder ao questionário (idosos com deficiência cognitiva severa, por exemplo), a informação foi obtida através do familiar presente no dia de visita.

\section{Amostra}

Foram entrevistados 484 indivíduos, divididos em 169 casos e 315 controles, em 1998-1999. O número de controles não corresponde ao dobro do número de casos, como seria esperado, porque algumas tríades não foram completadas, isto é, para alguns casos só foi possível obter um controle que preenchesse os critérios de pareamento.

\section{Variáveis estudadas}

A variável dependente foi internação hospitalar por fratura (grave) decorrente de queda. A variável independente foi o uso de medicamentos nas 24 horas anteriores à admissão no hospital.

\section{Análise dos dados}

Através de uma análise descritiva, foram levantadas as circunstâncias mais comuns envolvendo as quedas que levaram à hospitalização dos idosos. A associação entre o uso de medicamentos e quedas seguidas de fraturas graves, foi estimada através do cálculo de odds ratios (OR), para dados pareados e seus respectivos intervalos de confiança de $95 \%$. A presença de confusão ("confounding") foi investigada através de modelos logísticos (regressão logística condicional), comparando-se a magnitude dos ORs para a associação medicamento-fratura obtida nesses modelos, com e sem a inclusão de co-variáveis. Diferenças superiores a $20 \%$ entre o OR bruto e o OR ajustado, foram consideradas como critério para a presença de confusão. No caso dessa diferença ser inferior a $20 \%$, optou-se pelo modelo com maior precisão (menor intervalo de confiança). Os dados foram analisados através do programa Stata 7.0 (Stata Corporation, 2001).

\section{Resultados}

\section{Perfil das quedas}

Dentre os 169 casos de fratura decorrente de queda, $131(77,5 \%)$ eram do sexo feminino. A maioria das quedas ocorreu entre 6 horas da manhã e 6 da tarde (Tabela 1), sendo a menor freqüência observada durante a madrugada. Não foram encontradas diferenças estatisticamente significativas, quando se investigou o período da queda segundo sexo ou faixa etária. Embora dois terços das quedas tenham ocorrido na própria casa do idoso, os homens caíram mais na rua do que as mulheres ( $44,7 \%$ vs. $22,8 \%)$.

A proporção de idosos que caíram dentro de casa aumentou com a faixa etária, sendo de $58 \%$ para os de até 69 anos, $62,9 \%$ para aqueles entre 70 e 79 anos e $84,6 \%$ para os que têm 80 anos ou mais. Dentre aqueles que se acidentaram em casa, os homens caíram mais em seu próprio quarto (40\%), enquanto as mulheres tenderam a cair igualmente, nos diferentes cômodos da casa. Apenas $1 / 4$ de todos os que caíram referiram ter tropeçado em algo. Um terço disse que a superfície estava molhada.

\section{Características de casos e controles}

Casos e controles foram pareados por sexo, idade e hospital. Na Tabela 2, apresenta-se a distribuição de freqüência de algumas variáveis entre casos e controles. A pequena diferença na proporção de homens e mulheres nos dois grupos, deve-se ao fato de que, para alguns casos, só foi possível obter um controle que preenchesse os critérios de pareamento. A média de idade nos casos foi de 70,7 (ep = 0,39) e 71,9 (ep = 0,57) nos controles.

Havia uma proporção maior de casados entre os controles do que entre os casos. Isso pode explicar, pelo menos em parte, a menor pro-

Tabela 1

Distribuição das quedas durante as 24 horas.

\begin{tabular}{lrr}
\hline Período & $\mathbf{n}$ & \multicolumn{1}{c}{$\%$} \\
\hline 6-12 horas & 55 & 34,0 \\
12-18 horas & 53 & 32,7 \\
18-24 horas & 42 & 25,9 \\
24-6 horas & 12 & 7,4 \\
Total & 162 & 100,0 \\
\hline
\end{tabular}

Nota: 7 casos sem informação. 
Tabela 2

Características de casos e controles.

\begin{tabular}{|c|c|c|c|c|}
\hline \multirow[t]{2}{*}{ Variável } & \multicolumn{2}{|c|}{ Casos } & \multicolumn{2}{|c|}{ Controles } \\
\hline & $\mathrm{n}$ & $\%$ & $\mathrm{n}$ & $\%$ \\
\hline \multicolumn{5}{|l|}{ Sexo } \\
\hline Masculino & 38 & 22,5 & 80 & 25,4 \\
\hline Feminino & 131 & 77,5 & 235 & 74,6 \\
\hline \multicolumn{5}{|l|}{ Situação conjugal } \\
\hline Casado & 45 & 26,6 & 147 & 46,7 \\
\hline Viúvo & 79 & 46,7 & 127 & 40,3 \\
\hline Separado & 18 & 10,7 & 24 & 7,6 \\
\hline Solteiro & 27 & 16,0 & 17 & 5,4 \\
\hline \multicolumn{5}{|l|}{ Mora só } \\
\hline Sim & 30 & 18,0 & 35 & 11,2 \\
\hline Não & 137 & 82,0 & 277 & 88,8 \\
\hline \multicolumn{5}{|l|}{ Escolaridade } \\
\hline Primário incompleto & 54 & 38,3 & 130 & 48,3 \\
\hline Primário & 56 & 39,7 & 95 & 35,3 \\
\hline Ginásio & 18 & 12,8 & 21 & 7,8 \\
\hline 2o e 3 o graus & 13 & 9,2 & 23 & 8,6 \\
\hline \multicolumn{5}{|l|}{ Trabalha } \\
\hline Sim & 23 & 13,6 & 56 & 17,8 \\
\hline Não & 146 & 86,4 & 259 & 82,2 \\
\hline \multicolumn{5}{|c|}{ Auto-avaliação da saúde } \\
\hline Excelente & 14 & 8,8 & 22 & 7,1 \\
\hline Boa & 101 & 63,1 & 183 & 58,8 \\
\hline Razoável & 41 & 25,6 & 98 & 31,5 \\
\hline Ruim & 4 & 2,5 & 8 & 2,6 \\
\hline \multicolumn{5}{|l|}{ Demência } \\
\hline Sim & 56 & 34,6 & 79 & 25,3 \\
\hline Não & 106 & 65,4 & 233 & 74,7 \\
\hline \multicolumn{5}{|l|}{ Queda no último caso } \\
\hline Sim & 79 & 46,7 & 109 & 34,7 \\
\hline Não & 90 & 53,3 & 205 & 65,3 \\
\hline \multicolumn{5}{|l|}{ Fratura no último ano } \\
\hline Sim & 22 & 13,2 & 20 & 6,5 \\
\hline Não & 145 & 86,8 & 288 & 93,5 \\
\hline \multicolumn{5}{|l|}{ Tabagismo } \\
\hline Sim & 33 & 19,5 & 40 & 12,7 \\
\hline Não & 136 & 80,5 & 275 & 87,3 \\
\hline \multicolumn{5}{|l|}{ Álcool } \\
\hline Sim & 49 & 29,0 & 74 & 23,5 \\
\hline Não & 120 & 71,0 & 241 & 76,5 \\
\hline \multicolumn{5}{|l|}{$\begin{array}{l}\text { Álcool (quatro horas } \\
\text { antes da internação) }\end{array}$} \\
\hline Sim & 11 & 6,5 & 8 & 2,5 \\
\hline Não & 158 & 93,5 & 301 & 97,5 \\
\hline
\end{tabular}

porção de idosos vivendo só entre os controles. Dentre os casos, $22 \%$ havia concluído o ginásio, contra $16,4 \%$ dos controles.

A maioria dos casos e controles classificou a sua saúde como boa. De acordo com o questionário BOAS, 1/3 dos casos e 1/4 dos controles foram classificados como possíveis casos de demência.
Setenta e nove casos $(46,7 \%)$ e 109 controles $(34,7 \%)$, relataram pelo menos uma queda no ano anterior. Entre os homens, essa proporção foi de $31,4 \%$ e entre as mulheres foi de $41,3 \%$. O grupo com 80 anos ou mais, referiu $64,1 \%$ de quedas nos últimos 12 meses, contra $36,7 \%$ e $33,5 \%$ daqueles com $60-69$ anos e 70 79 , respectivamente. Não houve diferença quanto ao sexo para proporção de fraturas no ano anterior $(8,8 \%)$, mas o grupo etário com $80 \mathrm{ou}$ mais anos, apresentou maior freqüência de fraturas $-17,7 \%$ contra $7 \%$ e $8,1 \%$ nas outras duas faixas etárias.

Controles mostraram-se mais autônomos do que os casos nas tarefas da vida cotidiana relacionadas à alimentação, cuidado corporal e locomoção. Seis e meio porcento dos casos referiram ingestão de álcool até quatro horas antes da internação, contra $2,5 \%$ dos controles. Não foram identificadas diferenças entre casos e controles quanto ao uso de café, chá e refrigerantes.

\section{Variáveis potenciais de confusão}

Para selecionar as variáveis potenciais de confusão, utilizou-se dois critérios: (a) o modelo apresentado na Figura 1 e (b) a associação observada nos dados da co-variável com a ocorrência de queda/fratura, assim como a sua associação com o uso de medicamentos (entre os controles). Para esse segundo critério, utilizouse um nível de significância de 0,25 no teste da razão de verossimilhança para considerar a presença de tais associações. As variáveis selecionadas segundo este critério estão na Tabela 3. Embora as variáveis "auto-avaliação da saúde" e "derrame" não tenham preenchido este critério ( $p=0,76$ e 0,28 , respectivamente), elas foram consideradas na análise como variáveis potenciais de confusão em função do modelo teórico. Apesar de não constar da Tabela 3, o uso de medicamentos foi tratado com fator potencial de confusão para investigar a associação entre certos remédios e a ocorrência de fratura por queda. Por exemplo, o bloqueador de canal de cálcio e diuréticos foram considerados fatores potenciais de confusão um para o outro, na medida em que ambos eram fatores de risco para as quedas e seu uso aparecia associado. O mesmo ocorreu para o uso de digitálicos e diuréticos.

\section{Uso de medicamentos}

Na Tabela 4, são apresentados os odds ratios não ajustados e respectivos intervalos de confiança de $95 \%$, para a associação entre o uso de 


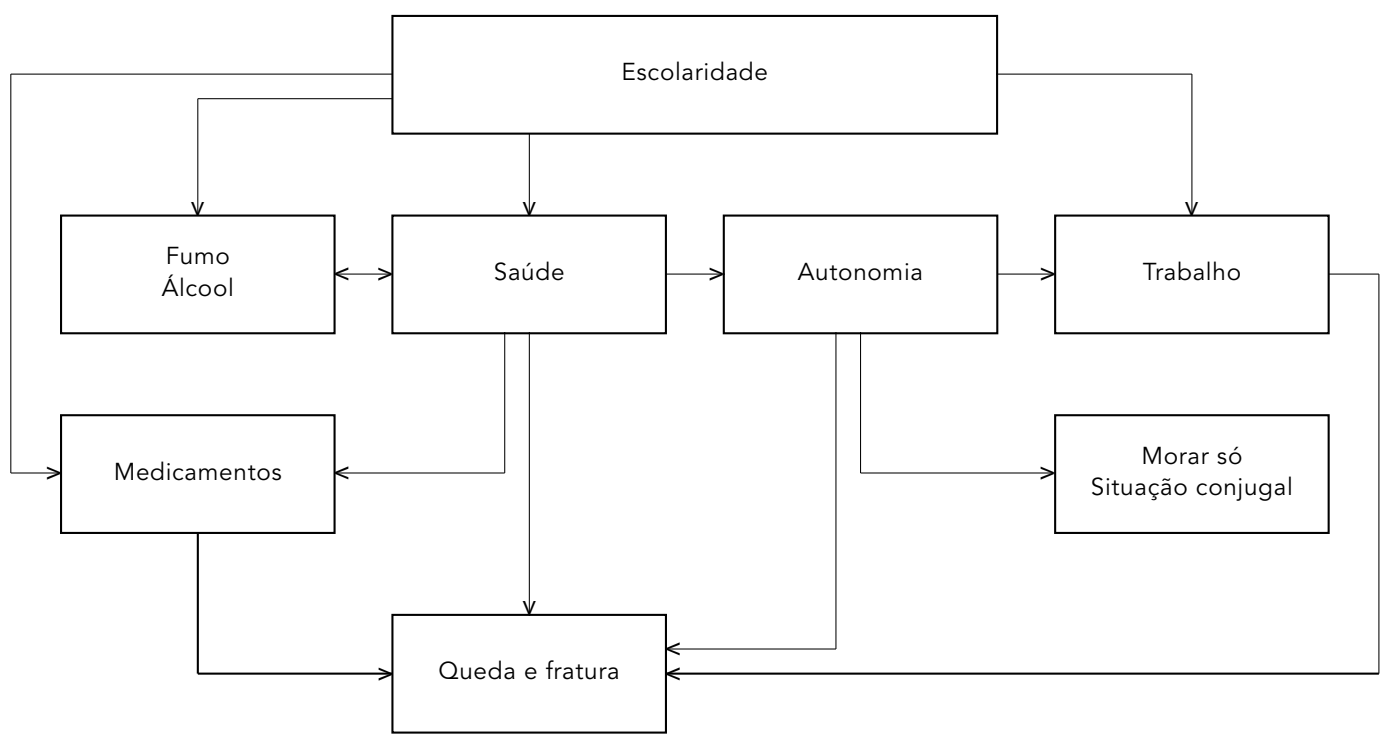

medicamentos nas últimas 24 horas e fratura decorrente de queda. Drogas bloqueadoras do canal de cálcio, benzodiazepínicos e vasodilatadores cerebrais associaram-se a um aumento no risco, enquanto os diuréticos associaram-se a uma redução desses acidentes. Miorrelaxantes e digitálicos associaram-se, em níveis limítrofes de significância estatística, ao aumento e redução, respectivamente, do risco.

Os medicamentos cuja associação apresentou um valor de $\mathrm{p}$ inferior a 0,25 , foram avaliados através de modelos logísticos condicionais para controle de variáveis potenciais de confusão. No caso das fraturas graves, os ORs podem ser interpretados tanto como uma razão de riscos quanto uma razão de taxas, já que a incidência esperada desses acidentes é de cerca de $5 \%$. Na Tabela 5, são apresentados os ORs e respectivos intervalos de confiança de $95 \%$ para os medicamentos cuja associação, após o controle por variáveis de confusão, apresentaram valor de p inferior a 0,10. De acordo com esses dados, houve um aumento no risco de fraturas graves por quedas de cerca de $96 \%$ entre os usuários de bloqueadores de canal de cálcio, e de $109 \%$ entre os idosos que usavam benzodiazepínicos. Já entre aqueles que faziam uso de diuréticos, o risco desses acidentes reduziu-se em cerca de $60 \%$. Há uma sugestão de que os usuários de antiácidos, digitálicos e laxantes teriam um risco menor de quedas seguidas de fraturas graves.

\section{Discussão}

Para alguns medicamentos, os resultados deste estudo estão de acordo com aqueles observados em investigações realizadas em outros países. Benzodiazepínicos têm sido associados a um aumento no risco de quedas e fraturas em diversos estudos individuais (Grisso et al., 1991; Jensen et al., 1991; Ray et al., 1987, 1989; Ryynänen, 1993), assim como numa meta-análise (Leipzig et al., 1999a).

$\mathrm{O}$ aumento no risco de quedas e fraturas entre idosos usuários de benzodiazepínicos, tem sido atribuído a duas propriedades desses medicamentos: atividade sedativa e bloqueio $\alpha$-adrenérgico. A primeira seria responsável por alterações psicomotoras, enquanto a segunda aumentaria a probabilidade de hipotensão postural. Os agentes hipnótico-sedativos de meia-vida longa, quando utilizados em doses clinicamente efetivas, podem causar sedação residual durante o dia entre os idosos. Com isso, esses indivíduos estão mais sujeitos a apresentar tonteiras, ataxia, confusão, levando ao risco aumentado de quedas. A redução da massa muscular e da densidade óssea acarreta uma 
Tabela 3

Variáveis associadas com fraturas decorrentes de quedas.

\begin{tabular}{|c|c|c|}
\hline Variável & Odds ratio & p-valor \\
\hline Situação conjugal & & $<0,001$ \\
\hline Casado & 1 & \\
\hline Viúvo & 2,30 & \\
\hline Separado & 3,08 & \\
\hline Solteiro & 6,60 & \\
\hline Escolaridade & & 0,25 \\
\hline Primário incompleto & 1 & \\
\hline Primário & 1,39 & \\
\hline Ginásio & 2,12 & \\
\hline 2o grau & 1,22 & \\
\hline 3o grau & 3,46 & \\
\hline Mora só & & 0,04 \\
\hline Sim & 1,79 & \\
\hline Não & 1 & \\
\hline Trabalha & & 0,22 \\
\hline Sim & 1 & \\
\hline Não & 1,44 & \\
\hline Diabetes & & 0,12 \\
\hline $\operatorname{Sim}$ & 1 & \\
\hline Não & 1,50 & \\
\hline Parkinson & & 0,02 \\
\hline Sim & 9,1 & \\
\hline Não & 1 & \\
\hline Epilepsia & & 0,22 \\
\hline Sim & 3,0 & \\
\hline Não & 1 & \\
\hline Reumatismo & & 0,03 \\
\hline Sim & 1 & \\
\hline Não & 1,60 & \\
\hline Osteoporose & & 0,16 \\
\hline Sim & 1,54 & \\
\hline Não & 1 & \\
\hline Nível de autonomia & & 0,10 \\
\hline Alto & 1 & \\
\hline Baixo & 1,49 & \\
\hline Tabagismo & & 0,02 \\
\hline Sim & 1,92 & \\
\hline Não & 1 & \\
\hline Uso de álcool & & 0,13 \\
\hline Sim & 1,44 & \\
\hline Não & 1 & \\
\hline $\begin{array}{l}\text { Uso de álcool (quatro } \\
\text { horas antes da internação) }\end{array}$ & & 0,02 \\
\hline Sim & 3,2 & \\
\hline Não & 1 & \\
\hline Queda no ano anterior & & 0,01 \\
\hline $\operatorname{Sim}$ & 1,7 & \\
\hline Não & 1 & \\
\hline Demência & & 0,04 \\
\hline Sim & 1,6 & \\
\hline Não & 1 & \\
\hline
\end{tabular}

probabilidade aumentada de que tais quedas resultem em fraturas (Ray et al., 1987).

Os resultados que associam o uso de bloqueadores de canal de cálcio a quedas e fraturas entre idosos são inconsistentes. Da mesma maneira que em nosso estudo, Cumming et al. (1991) encontraram um risco aumentado desses acidentes entre usuários desses medicamentos. Embora associação referida por esses autores tenha perdido significância estatística após o controle por múltiplas variáveis de confusão, eles observaram uma relação positiva entre a dose e o risco de múltiplas quedas. Leipzig et al. (1999b) não observaram associação entre quedas e o uso desses medicamentos em sua meta-análise.

Segundo Oates \& Brown (2001), as drogas bloqueadoras de canal de cálcio como o verapamil, a nifedipina (ambas muito utilizadas entre idosos deste estudo), apresentam um efeito cardíaco inotrópico negativo, que é normalmente compensado através de um aumento da estimulação adrenérgica cardíaca para compensar a hipotensão. Em indivíduos idosos, esse reflexo costuma estar reduzido, o que pode levar ao maior risco de hipotensão, aumentando o risco de quedas.

Embora o presente estudo tenha identificado uma redução do risco de fraturas graves, decorrentes de quedas nos idosos que faziam uso de diuréticos, as investigações têm mostrado resultados controversos. Há referência à redução desse risco (LaCroix et al., 1990; Ray et al., 1989), ao aumento (Cumming et al., 1991; Prudham \& Evans, 1981) ou ausência de associação (Leipzig et al., 1999b). O possível efeito protetor dos diuréticos tiazídicos tem sido atribuído a uma redução na excreção urinária de cálcio, com conseqüente aumento da densidade óssea. Por outro lado, Cumming et al. (1991) atribuem o maior risco de quedas à hipocalemia, arritmias e hipotensão postural. Guo et al. (1998) sugerem que tal inconsistência possa decorrer de não se considerar o uso de suplementação de potássio nos estudos realizados até o momento. Esses autores observaram um efeito protetor dos diuréticos não tiazídicos, que desapareceu com a inclusão de potássio no modelo.

O uso de antiácidos e digitálicos mostrouse associado ao menor risco de hospitalização por fratura decorrente de queda, embora com níveis de significância estatística limítrofes $(0,05$ $<\mathrm{p}<0,10$ ). Esses achados não foram reportados por nenhum dos estudos revisados, e são de difícil interpretação. Ao contrário da proteção sugerida por nossos dados através de uma associação com significância estatística limítrofe, Cumming et al. (1991) encontraram um 
aumento no risco de múltiplas quedas associado ao uso de laxantes, que atribuíram a um possível viés de memória. Em nosso estudo, a maior freqüência desses medicamentos entre os controles poderia ser atribuída a um viés de seleção, onde os controles hospitalares teriam maior uso desse medicamento nas 24 horas anteriores à internação, em decorrência de preparo para procedimentos cirúrgicos. Entretanto, quando se repetiu a análise considerando-se apenas o uso durante as duas últimas semanas (excluindo-se as 24 horas antes da internação), o resultado se manteve.

Dentre os aspectos metodológicos do presente estudo que podem ter afetado seus resultados, devem ser considerados os viéses de seleção e de memória. No caso do primeiro, o uso de controles hospitalares pode ter contribuído para que a exposição aos medicamentos, observadas entre os controles, não correspondesse àquela esperada na base populacional de onde vieram os casos. Em que pese o cuidado em selecionar doenças que, possivelmente, não estavam associadas com os medicamentos em estudo, não há garantia de que tal procedimento tenha sido bem sucedido. Por outro lado, a presença de cirurgias eletivas no grupo de controles, pode ter gerado um conjunto de idosos mais sadios nesse grupo. Embora se tenha tentado contornar esse problema, incluindo variáveis de estado de saúde e doenças específicas, estas foram auto-referidas e não o resultado de um exame clínico-laboratorial (exceto para demência). Quanto ao viés de memória, um estudo sobre a confiabilidade da informação sobre o uso de medicamentos, realizado no início desta pesquisa (Coutinho et al., 1999), mostrou medidas elevadas de confiabilidade, embora motivos operacionais não tenham permitido a realização de um estudo de validade.

$\mathrm{O}$ avanço no entendimento do papel do uso de medicamentos nas fraturas graves entre idosos, passa pela realização de estudos similares a este, assim como a utilização de controles da população em geral nos estudos casocontrole, e a realização de estudos de coortes para lidar com os viéses de memória e de seleção. A investigação da interação entre esses medicamentos e fatores demográficos e sócioeconômicos, também é de extrema importância na compreensão e prevenção desse problema de saúde pública. Entretanto, desde já deve-se ressaltar a necessidade de ponderar os riscos e benefícios no uso de medicamentos em idosos, assim como orientar tais indivíduos e seus familiares para evitar esses acidentes, quando o uso de drogas associadas ao maior risco de quedas é necessário.

\begin{tabular}{|c|c|c|c|}
\hline Medicamento & $\begin{array}{l}\text { Odds ratio } \\
\text { não ajustado }\end{array}$ & $\begin{array}{l}\text { Intervalo de } \\
\text { confiança de } 95 \%\end{array}$ & p-valor \\
\hline Qualquer medicamento & 1,22 & $0,77-1,91$ & 0,39 \\
\hline Antiácidos & 0,44 & $0,16-1,22$ & 0,11 \\
\hline Antialérgicos & - & - & - \\
\hline Bloqueadores $\alpha$-adrenérgicos & 0,79 & $0,27-2,30$ & 0,66 \\
\hline Antidepressivos & 2,00 & $0,40-9,91$ & 0,40 \\
\hline Antigripais & 2,00 & $0,12-31,98$ & 0,62 \\
\hline Antiinflamatórios & 1,41 & $0,82-2,43$ & 0,21 \\
\hline Analgésicos & 0,88 & $0,58-1,34$ & 0,55 \\
\hline Bloqueadores $\beta$-adrenérgicos & 0,88 & $0,41-1,91$ & 0,75 \\
\hline Bloqueadores de canal de cálcio & 1,67 & $1,01-2,74$ & 0,04 \\
\hline Benzodiazepínicos & 2,27 & $1,20-4,31$ & 0,01 \\
\hline Cálcio & 1,50 & $0,34-6,70$ & 0,60 \\
\hline Colírios & 2,00 & $0,28-14,20$ & 0,49 \\
\hline Diuréticos & 0,54 & $0,28-1,01$ & 0,05 \\
\hline Digitálicos & 0,16 & $0,02-1,31$ & 0,09 \\
\hline Inibidores da ECA & 0,86 & $0,46-1,61$ & 0,64 \\
\hline Laxantes & 0,41 & $0,11-1,47$ & 0,17 \\
\hline Miorrelaxantes & 1,73 & $0,98-3,06$ & 0,06 \\
\hline Nitratos & 1,62 & $0,65-4,04$ & 0,30 \\
\hline Outros & 0,77 & $0,51-1,16$ & 0,21 \\
\hline Vasodilatadores periféricos & 1,00 & $00,9-11,02$ & 1,00 \\
\hline Vasodilatadores cerebrais & 2,79 & $0,98-7,91$ & 0,05 \\
\hline
\end{tabular}

Tabela 5

Associação entre medicamentos usados nas últimas 24 horas e fratura decorrente de quedas.

\begin{tabular}{lccc}
\hline Medicamento & $\begin{array}{l}\text { Odds ratio } \\
\text { ajustado }\end{array}$ & $\begin{array}{l}\text { Intervalo de } \\
\text { confiança de } 95 \%\end{array}$ & p-valor \\
\hline Antiácidos1 & 0,38 & $0,13-1,15$ & 0,09 \\
Bloqueadores de canal de cálcio2 & 1,96 & $1,16-3,30$ & 0,01 \\
Benzodiazepínicos3 & 2,09 & $1,08-4,05$ & 0,03 \\
Diuréticos4 & 0,40 & $0,20-0,80$ & 0,02 \\
Digitálicos5 & 0,16 & $0,02-1,31$ & 0,09 \\
Laxantes6 & 0,22 & $0,04-1,11$ & 0,07 \\
\hline
\end{tabular}

Ajustado por:

1 auto-avaliação da saúde, uso de antiinflamatórios, reumatismo.

2 auto-avaliação da saúde, uso de diuréticos.

3 auto-avaliação da saúde.

4 osteoporose, uso de bloqueador de canal de cálcio.

5 sem co-variáveis.

6 escolaridade, uso prévio de álcool. 


\section{Referências}

CHAIMOWICZ, F.; MARTINS, T. J. X. \& ASSUMPÇÃO, D. F., 2000. Use of psychoactive drugs and falls among older people living in a community in Brazil. Revista de Saúde Pública, 34:631-635.

COUTINHO, E. S. F.; SILVA, S. D.; PAIVA, C. H. A.; FREITAS, G. G.; MIRANDA, L. M. \& MENDONÇA, R., 1999. Confiabilidade da informação sobre o uso recente de medicamentos em um estudo caso-controle de base hospitalar. Cadernos de Saúde Pública, 15:553-558.

CUMMING, R. G.; MILLER, J. P.; KELSEY, J. L.; DAVIS, P.; ARFKEN, C. L.; BIRGE, S. J. \& PECK, W. A., 1991. Medications and multiple falls in elderly people: The St. Louis OASIS Study. Age and Aging, 20:455461.

GRISSO, J. A.; KELSEY, J. L.; STROM, B.; CHIU, G. Y.; MAISLIN, G.; O'BRIEN, L. A.; HOFFMAN, S. \& KAPLAN, F., 1991. Northeast Hip Fracture Study: Risk factors for falls as a cause of hip fracture in women. New England Journal of Medicine, 324: 1326-1331.

GUO, Z.; WILLS, P.; VITANEN, M.; FASTBOM, J. \& WINBLAD, B., 1998. Cognitive impairment, drug use, and the risk of hip fracture in persons over 75 years old: A community-based prospective study. American Journal of Epidemiology, 148:887-892.

JENSEN, J.; NIELSEN, L. H.; LYHNE, N.; HALLAS, J. \& BROSEN, K., 1991. Drugs and femoral neck fracture: A case-control study. Journal of Internal Medicine, 229:29-33.

KALACHE, A.; VERAS, R. P. \& RAMOS, L. R., 1987. O envelhecimento da população mundial. Um desafio novo. Revista de Saúde Pública, 21:200-210.

LACROIX, A. Z.; WIENPAHL, J.; WRITE, L. R.; WALLACE, R. B.; SCHERR, P. A.; GEORGE, L. K.; HUNTLEY, J. C. \& OSTFELD, A. M., 1990. Thiazide diuretic agents and the incidence of hip fracture. New England Journal of Medicine, 322:286-290.

LEIPZIG, R. M.; CUMMING, R. G. \& TINETTI, M. E., 1999a. Drugs and falls in older people: A systematic review and meta-analysis: I. Psychotropic drugs. Journal of the American Geriatric Society, 47:30-39.

LEIPZIG, R. M.; CUMMING, R. G. \& TINETTI, M. E., 1999b. Drugs and falls in older people: A systematic review and meta-analysis: II. Cardiac and analgesic drugs. Journal of the American Geriatric Society, 47:40-50.

MONTEIRO, M. F. G. \& ALVES, M. I. C., 1995. Aspectos demográficos da população idosa no Brasil. In: Terceira Idade: Um Envelhecimento Digno para o Cidadão do Futuro (R. Veras, org.), pp. 65-78, Rio de Janeiro: Relume-Dumará/Universidade Aberta da Terceira Idade, Universidade do Estado do Rio de Janeiro.
OATES, J. A. \& BROWN, N. J., 2001. Antihypertensive agents and the drug therapy of hypertension. In: Goodman \& Gilman's The Pharmacological Basis of Therapeutics (J. G. Hardman \& L. E. Limb, ed.), pp. 871-900, 10th Ed. New York: McGraw-Hill.

PINHEIRO, R. S., 1999. Estudo sobre Variações no Uso de Serviços de Saúde: Abordagens Metodológicas e a Utilização de Grandes Bases de Dados Nacionais. Tese de Doutorado, Rio de Janeiro: Escola Nacional de Saúde Pública, Fundação Oswaldo Cruz.

PRUDHAM, D. \& EVANS, J., 1981. Factors associated with falls in the elderly: A community study. Age and Ageing, 10:141-146.

RAY, W. A.; GRIFFIN, M. R. \& DOWNEY, W., 1989. Benzodiazepines of long and short elimination halflife and the risk of hip fracture. JAMA, 262:33033307.

RAY, W. A.; GRIFFIN, M. R.; SCHAFFNER, W.; BAUGH, D. K. \& MELTON, J., 1987. Psychotropic drug use and the risk of hip fracture. New England Journal of Medicine, 316:363-369.

ROZENFELD, S., 1997. Reações Adversas aos Medicamentos na Terceira Idade: As Quedas como Iatrogenia Farmacoterapêutica. Tese de Doutorado, Rio de Janeiro: Instituto de Medicina Social, Universidade do Estado do Rio de Janeiro.

RYYNÄNEN, O. P., 1993. Incidence and Risk Factors for Falling Injuries Among the Elderly. Acta Universitatis Ouluensis, Series D, v. 265. Oulu: University of Oulu.

RYYNÄNEN, O. P.; KIVELÄ, S. L.; HONKANEN, R.; LAIPPALA, P. \& SOINI, P., 1991. Incidence of falling injuries leading to medical treatment in the elderly. Public Health, 105:373-386.

SATTIN, R. W., 1992. Falls among older persons: A public health perspective. Annual Review of Public Health, 13:489-508.

STATA CORPORATION, 2001. Stata Statistical Software: Release 7.0. College Station: Stata Corporation.

TINETTI, M. E., 1994. Prevention of falls and fall injuries in elderly persons: A research agenda. Preventive Medicine, 23:756-762.

VERAS, R. P. \& COUTINHO, E. S. F., 1991. Estudos de prevalência de depressão e síndrome cerebral orgânica na população de idosos, Brasil. Revista de Saúde Pública, 25:209-217.

Recebido em 19 de outubro de 2001

Aprovado em 26 de março de 2002 